\title{
Fabricación de componentes constructivos con la fracción plástica de residuos provenientes del reciclado de RAEE
}

\section{Manufacture of construction components with plastic fraction of WEEE recycling waste}

Presentación: 6-7/10/2020

\section{Doctorando:}

\section{Melina Gabriela Gómez}

Nuevos Materiales, Instituto de Física Aplicada, Universidad Nacional de San Luis - Argentina dimelinag@gmail.com

\section{Director/es:}

\section{Dr. Jerónimo Kreiker}

Co-director/es:

\section{Dr. Lucas Ernesto Peisino}

Dra. Bárbara Belén Raggioti

\begin{abstract}
Resumen
Los residuos de aparatos eléctricos y electrónicos (RAEE), han crecido notablemente en cantidad en los últimos años y se espera que esta tendencia continúe. La fracción plástica del desecho (FPR), constituye aproximadamente el $30 \%$ del total en peso, y no ha podido ser insertada en el circuito del reciclado, debido a la heterogeneidad de plásticos constitutivos y a la presencia de contaminantes, principalmente retardantes de llama y metales pesados, ambos tóxicos para la salud humana.

Existen numerosos estudios sobre el agregado de plásticos a morteros, actuando como material de carga o fibras de refuerzo, logrando materiales con aptitud para este uso. El estudio de estos antecedentes llevó a considerar el uso de la FPR como agregado en morteros, en reemplazo total y parcial del agregado tradicional.

Se desarrolló un agregado sintético (AS) mediante la estrategia del core-shell, en el cual el núcleo de plástico es recubierto con sucesivas capas de cemento y se estudió su desempeño como componente constructivo mediante ensayos de resistencia mecánica y determinación de propiedades físicas. El AS puede ser utilizado en morteros en reemplazo total y parcial de agregado fino, sin disminuir significativamente la resistencia mecánica. A pesar de los buenos resultados que se obtuvieron, con el paso del tiempo aparecen exudados amarillos en la superficie resultado de la desestabilización de los retardantes de llama en el medio básico del cemento, lo cual lo hace potencialmente peligroso para el uso en construcciones civiles. Para revertir este efecto se agregó al proceso carbón activado (CA) como aditivo estabilizante. Este trabajo presenta una síntesis de los resultados expuestos en las Jornadas 2019 y el desarrollo de lo realizado durante el corriente año. Se exhiben los resultados del ensayo de succión capilar con el uso de diferentes tipos y dosificaciones de CA, y un Análisis de Ciclo de Vida (ACV) preliminar del impacto durante su etapa de fabricación.
\end{abstract}

Palabras clave: agregado sintético, plástico de RAEE, mortero sustentable.

\footnotetext{
Abstract

Waste electrical and electronic equipment (WEEE) has grown notably in quantity in recent years and this trend is expected to continue. The plastic fraction of the waste (FPR), constitutes approximately $30 \%$ of the total by weight, and has not been able to be inserted into the recycling circuit, due to the heterogeneity of constituent plastics and the presence of contaminants, mainly flame retardants and metals. heavy, both toxic to human health.

There are numerous studies on the addition of plastics to mortars, acting as filler or reinforcing fibers, achieving materials suitable for this use. The study of these antecedents led to consider the use of RPF as an aggregate in mortars, in total and partial replacement of the traditional aggregate.
} 
A synthetic aggregate (AS) was developed through the core-shell strategy, in which the plastic core is covered with successive layers of cement and its performance as a constructive component was studied through mechanical resistance tests and determination of physical properties. AS can be used in mortars in total and partial replacement of fine aggregate, without significantly reducing the mechanical resistance. Despite the good results obtained, over time, yellow exudates appear on the surface as a result of the destabilization of the flame retardants in the basic cement medium, which makes it potentially dangerous for use in civil constructions. To reverse this effect, activated carbon (AC) was added to the process as a stabilizing additive. This work presents a synthesis of the results presented in the 2019 Conference and the development of what has been done during the current year. The results of the capillary suction test are exhibited with the use of different types and dosages of AC, and a preliminary Life Cycle Analysis (LCA) of the impact during its manufacturing stage.

Keywords: synthetic aggregate, WEEE plastic, sustainable mortar.

\section{Introducción}

Los plásticos no pueden degradarse de forma natural como los residuos orgánicos, quedan en la naturaleza por cientos de años alcanzando a nivel mundial 150.000.000 toneladas anuales (Singh et al., 2017). El caso de los residuos de aparatos eléctricos y electrónicos (RAEE), es uno de los más preocupantes debido al incremento que ha sufrido en las últimas dos décadas ("Glob. Waste Manag. Outlook," 2016) y porque posee características comunes al resto de los plásticos, como su baja bio-degradabilidad que hace que se acumule en los basurales obstruyendo los procesos naturales del agua y la tierra y características únicas, como la presencia de metales pesados como el cadmio (Cd) que se emplea como pigmento, estabilizador o catalizador durante el proceso de polimerización de, por ejemplo el ABS (Dimitrakakis et al., 2009; Tamaddon, F., \& Hogland, 1993) y otros como el Al, $\mathrm{Ca}, \mathrm{Cu}, \mathrm{Fe}, \mathrm{Mg}, \mathrm{Ni}, \mathrm{Pb}$ y $\mathrm{Zn}$ en distintas concentraciones (Stenvall et al., 2013) y de sustancias químicas tóxicas persistentes que contaminan el medio ambiente y afectan a la salud de las personas (Greenpeace, 2011). La presencia de estos contaminantes en los artefactos eléctricos y electrónicos (AEE) los tornan más contaminantes para el ambiente que cualquier otro residuo sólido. Al ser dispuestos en un basural sin tratamiento previo, los metales pesados y otros compuestos que suelen contener, migran mediante procesos de lixiviación hacia las napas de agua y al ambiente (Devia, 2012). Resultan peligrosos también para los trabajadores de las plantas de gestión de RAEE, que están expuestos al manejo de ciertas sustancias o desechos que pueden ser riesgosos para su salud y el entorno ambiental.

La fracción plástica de los RAEE (FPR) forma, en promedio un 34\% del AEE, siendo los componentes electrónicos y los metales los que le siguen en porcentaje (Dimitrakakis et al., 2009). Otra particularidad de esta fracción plástica es su composición heterogénea; está formada por mezclas de: ABS/PC en un 5\%; PMMA: 3\%; PC: 3\%; PA: 1\%; ABS: 29\%; HIPS: 26\%; PP: 22\%; y otros polímeros, $11 \%$. Mientras que los aditivos y cargas que contiene son: fósforo, cloro, antimonio, bromo, cromo, cadmio, plomo, óxido de magnesio, alúmina, silicato, carbonato de calcio y titanio (Maris et al., 2015) .Esta característica hace que su reciclado sea aún más complejo debido a que, para la re-extrusión se requiere un sólo tipo de plástico en la muestra(Unnisa \& Hassanpour, 2017) al mismo tiempo que los procesos de separación requerirían un costo extra correspondiente a la incorporación de pasos en la cadena de producción, como la selección manual, disolución selectiva, flotación, fluorescencia de rayos X, espectroscopia láser, entre otros (Unnisa \& Hassanpour, 2017).

Por otro lado, la FPR también por estar en contacto con circuitos electrónicos, debe cumplir con ciertas normas de seguridad establecidas para evitar la propagación de incendios, para esto se utilizan sustancias químicas que se agregan o reaccionan con materiales combustibles para aumentar su resistencia al fuego: los retardantes de llama bromados (BFR's). Existen actualmente 75 tipos de BFRs disponibles en el mercado, siendo los más comunes y prioritarios los polibromodifeniléteres incluyendo mezclas comerciales de PentaBDE, OctaBDE y DecaBDE, el tetrabromobisfenol A (TBBPA) y el hexabromociclododecano (HBCD) (Aldrian et al., 2015) (Informe, A. y Ta-, 2008) y se aplican en gran cantidad de materiales que requieran de resistencia al fuego, pero es en los aparatos eléctricos y electrónicos donde junto al óxido de antimonio (Sb203) como sinérgico (Aldrian et al., 2015) en el HIPS y ABS, que se presentan con mayor porcentaje (56\%). El TBBA es el BFR que usa en la mayoría de los RAEE (Unnisa \& Hassanpour, 2017). Es un polvo cristalino blanco (o incoloro), que contiene un 50\% de bromo (Informe, A. y Ta-, 2008). Si bien los retardantes de llama son una solución a la propagación del fuego, pueden salvar vidas y prevenir daños materiales, también son objeto de una creciente preocupación sobre sus impactos medioambientales y efectos sobre la salud ya que no son adecuadamente tratados en la actualidad por el sector informal generando riesgos tanto para los trabajadores como para los consumidores (Haarman \& Gasser, 2016). Algunos de ellos ya han sido probados como perjudiciales para el medio ambiente o los seres humanos, y se ha cesado su producción (CARPL, 2010). Es necesario entonces, llevar a cabo un proceso de reciclaje que considere la existencia de componentes metálicos, aditivos RAEE contiene.

En esta tesis se propone: valorizar el plástico de RAEE reinsertándolo en un ciclo productivo y a su vez, neutralizando la exposición de sus componentes metálicos y contaminantes orgánicos. Se aplicarán estos métodos con 
el objetivo de generar materiales con recursos recuperados y combinados para ser empleados en componentes constructivos o en construcción de manera directa. La incorporación de residuos plásticos en materiales de construcción es una buena alternativa para reducir tal desecho. Con este método los plásticos se reaprovechan sin perder calidad y se sustituyen por el uso de los materiales vírgenes en la construcción (Schlummer, M., Mäurer, A., Leitner, T., \& Spruzina, 2006). Mediante la técnica de core-shell (corazón-coraza) el plástico se cubre con capas sucesivas de cemento de albañilería y carbón activado. El cemento actúa como estabilizante de metales mientras que el carbón activado como estabilizante de BFR's.

\section{Desarrollo}

Se desarrolló un agregado de plástico reciclado (APR) que consiste en partículas de plástico de RAEE (FPR) trituradas recubiertas con 3 capas de una mezcla de cemento y carbón activado para ser empleadas en la industria de la construcción como reemplazo total o parcial del agregado fino y grueso natural. Se obtuvo plástico proveniente del RAEE de varios proveedores locales, quienes lo entregan triturado y mezclado en tamaños de partículas de entre 3-8 mm. Para la fabricación, las partículas se pesan y se introducen en la máquina mezcladora vertical sin paletas. El cemento y el carbón activado se dosifican y se mezclan previamente. Luego, se incorporan a la mezcladora, donde ya están las partículas y se va agregando el agua hasta completar una relación agua/cemento 0,5. Las partículas se dejan secar 24 horas. Luego de la aplicación de cada capa se realiza un desterronado y tamizado. El APR se puede utilizar a partir de los 7 días, aunque se recomienda su uso recién después de 28 días, lapso considerado óptimo para el fraguado del cemento.
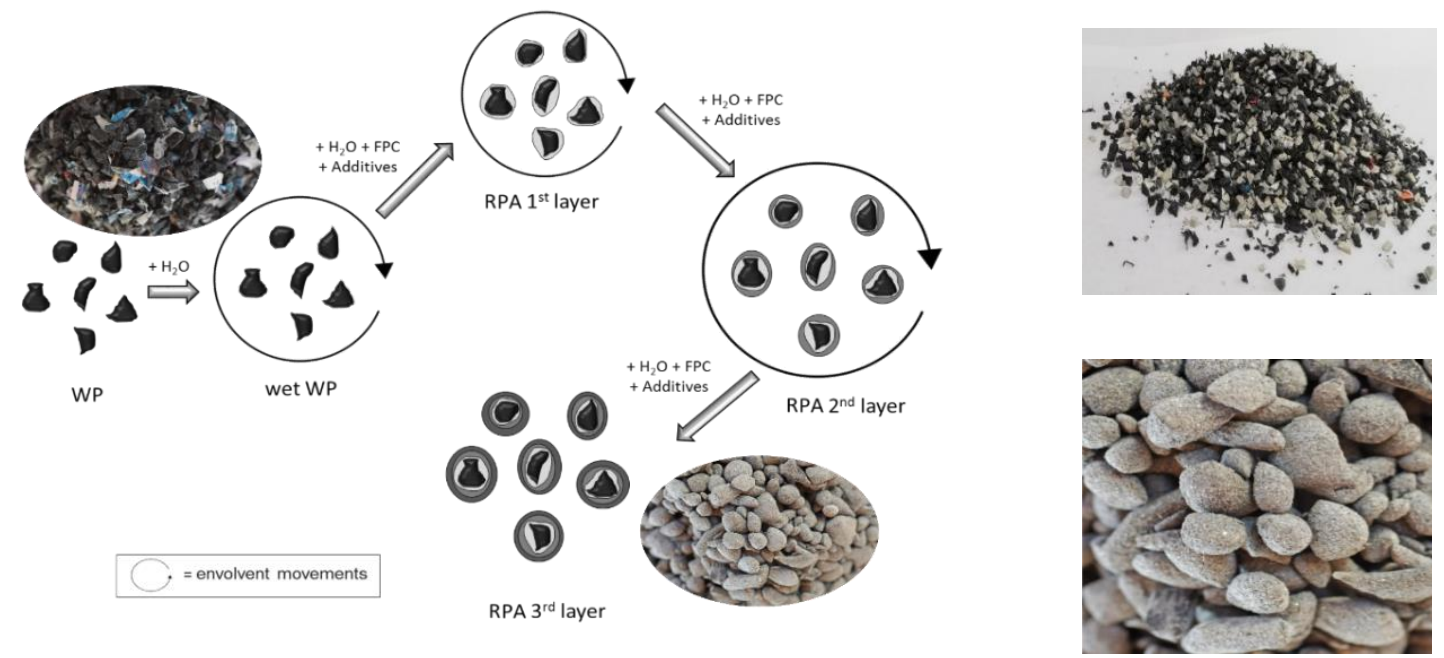

Fig. 1: Proceso de fabricación de APR. a) FPR. b) APR elaborado por la técnica del Core-Shell

Se elaboraron muestras con diferente dosificación FPR/cemento+aditivos, algunas con presencia de carbón activado y otras sin él, con el objetivo de evaluar el comportamiento mecánico y las características de las distintas combinaciones. Otras muestras consistieron en la FPR triturada a dos granulometrías diferentes sin ningún tipo de recubrimiento. En la tabla 1 se detalla la composición de cada muestra.

\begin{tabular}{|c|c|c|c|c|c|}
\hline Entrada & Muestra & $\begin{array}{c}\text { Plástico de RAEE } \\
\text { (\% en peso) }\end{array}$ & $\begin{array}{c}\text { Cemento de albañilería } \\
\text { (\% en peso) }\end{array}$ & $\begin{array}{c}\text { Carbón activado } \\
\text { (tipo-\% en peso) }\end{array}$ & $\begin{array}{c}\text { Tamaño } \\
\text { (mm) }\end{array}$ \\
\hline 1 & A & 43 & 52 & CAE-061 - 5 & 3 \\
\hline 2 & B & 44 & 53 & CAE-061 - 3 & 3 \\
\hline 3 & C & 43 & 52 & CAE U - 5 & 8 \\
\hline 4 & D & 44 & 53 & CAE U - 5 & 3 \\
\hline 5 & E & 44 & 53 & & 8 \\
\hline 6 & F & 45 & 55 & & 3 \\
\hline 7 & G & 45 & 55 & & 8 \\
\hline 8 & RAEE 3 & 100 & -- & & 3 \\
\hline 9 & RAEE 8 & 100 & -- & & 8 \\
\hline
\end{tabular}

Tabla 1: Composición de las distintas muestras realizadas 
Sobre los APR desarrollados se estudiaron propiedades como granulometría, densidad aparente, forma y ensayos químicos para determinar liberación de metales y aditivos ignífugos. Para determinar su comportamiento como agregado se elaboraron probetas con cemento y APR y se realizaron ensayos de resistencia mecánica a la compresión y flexión. Resultados que fueron presentados en seminarios previos. Los nuevos resultados corresponden a los ensayos desarrollados durante este año de succión capilar y se incorpora también un ACV preliminar para la etapa de fabricación.

Succión capilar: con respecto a la capacidad y velocidad de succión de agua mediante capilares, se desarrolló el ensayo bajo la norma IRAM 1871. Se realizaron ensayos para todas las probetas; en la figura 1 se exponen las capacidades y velocidades de succión capilar en cuanto a composición APR. La capacidad está dada por el valor correspondiente al extremo final de la curva y la velocidad, o sea la rapidez con que ingresa el líquido por los poros de hormigón, está dada por la pendiente de la recta obtenida al graficar el incremento de masa por unidad de área en gramos por metro cuadrado. La probeta $\mathrm{D}$, que contiene un $5 \%$ de CA del tipo CAE U, presentó la mayor capacidad y velocidad de succión capilar, esto debido a que este tipo de CA se fabrica a base de madera y posee un $n^{\circ}$ de yodo de 1050,35 mg. El número de yodo es el cálculo de los miligramos de yodo que puede absorber por gramo de carbón; es una medida de la porosidad que determina la menor o mayor capacidad de succión, a mayor número de yodo mayor será la capacidad de succión. Es por esto también que la probeta A tiene un valor significativamente menor de capacidad de succión que esta última, el $\mathrm{n}^{\circ}$ de yodo del tipo de CA que contiene tiene un valor de $800 \mathrm{mg}$. Entre estos valores, se encuentran los de la probeta F, que no contiene CA. Presenta un valor significativamente menor que " $\mathrm{D}$ ". Esto podría deberse a la ausencia del efecto adsorbente que presenta el carbón activado, en mayor o menor medida según el tipo o fabricante.

La probeta que se fabricó incorporando partículas de RAEE triturado directamente a la mezcla de cemento presentó un comportamiento similar a " $\mathrm{F}$ ", aquí se observa una particularidad en relación a la velocidad, si bien, son similares los valores, RAEE3 presenta una gran velocidad de succión durante las primeras horas que luego se ralentiza igualando a las demás curvas; esto se debe a que en las primeras horas es el cemento el que absorbe el agua y luego esta se encuentra con la superficie hidrofóbica del plástico que hace que la curva de aplane.

La probeta patrón presentó valores aceptables para el reglamento CIRSOC 201-05 pero las probetas de APR, con o sin carbón activado presentaron valores excesivamente altos. Cabe destacar que, en todos los casos los valores de velocidad corresponden a los de capacidad; a mayor capacidad, mayor velocidad.

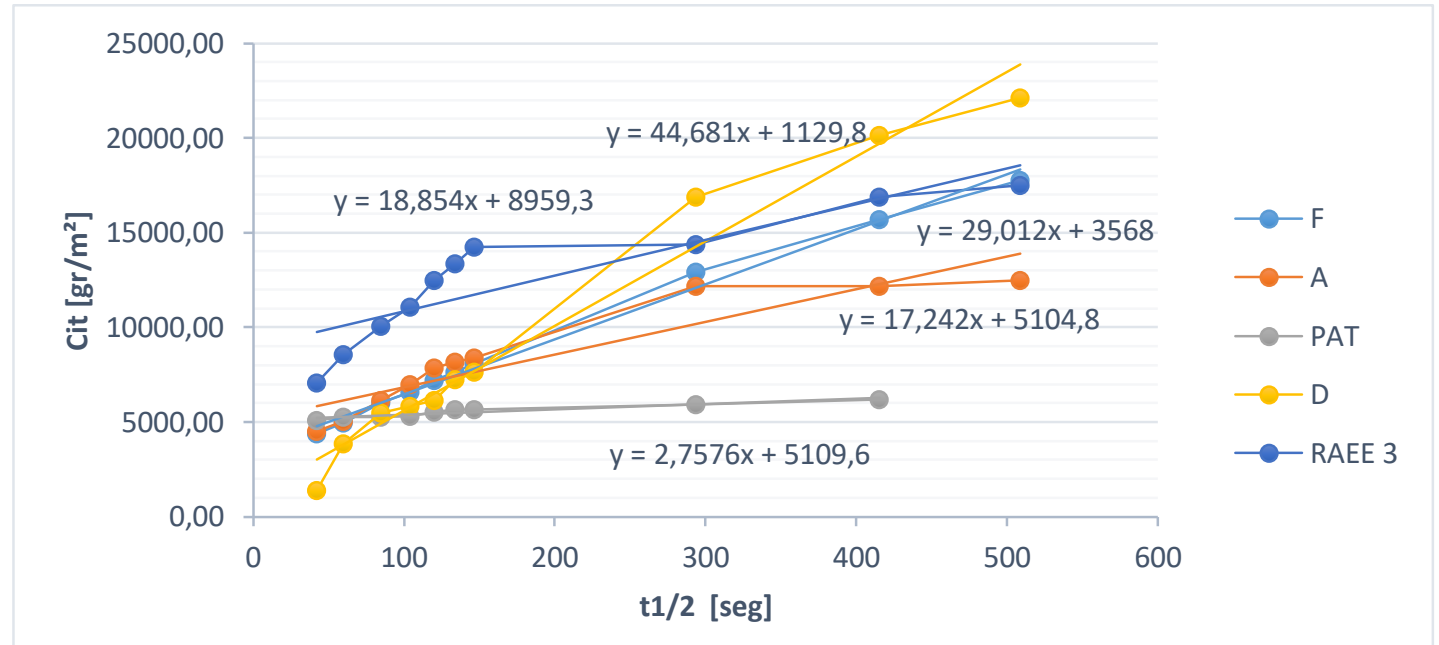

Figura 2: capacidad y velocidad de succión capilar de las probetas de granulometría $3 \mathrm{~mm}$.

Con respecto al tamaño de APR, los resultados sugieren que el tamaño del árido interfiere en la capacidad de succión del mortero. Tanto la capacidad como la velocidad de succión capilar disminuyen al aumentar el tamaño del agregado esto se debe a la presencia de aire y cemento en los intersticios de la probeta generados por la falta de un árido de menor tamaño que los ocupe. La presencia de burbujas de aire, por aire naturalmente incorporado puede tener su origen en el estado plástico debido al fenómeno de exudación por medio del cual se generan canales capilares que constituyen una red de poros interconectados que generan que el ascenso de agua se interrumpa por la presencia de un agregado grueso y permanecer debajo de este para evaporase durante el secado. Esto no necesariamente reviste un beneficio ya que de esta manera aparecen oquedades debajo de los agregados que disminuyen su resistencia, durabilidad mientras que provee una vía de acceso directa a los agentes externos (Taus, 2010). 
Análisis de Ciclo de Vida: se desarrolló un análisis del ciclo de vida (ACV) preliminar del APR abarca desde el ingreso de la materia prima a la planta de fabricación hasta la obtención del componente final (de la puerta a la puerta). La unidad funcional seleccionada es de $1 \mathrm{t}$ de APR y las categorías de impacto ambiental consideradas son el Consumo Energético (CE) y calentamiento global por gases de efecto invernadero (GEI) que dicho consumo genera (CO2, $\mathrm{CH} 4$, N20) El objetivo es obtener el valor del consumo acumulado de energía (CAE), en el proceso de fabricación del APR y la cantidad de emisiones de calentamiento global (GEI) que produce tal consumo.

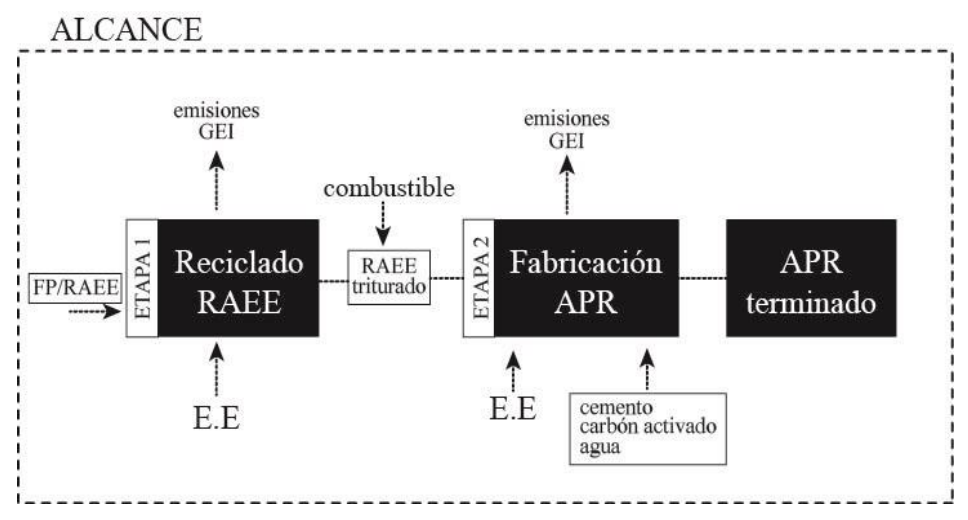

Figura 3: Gráfico del alcance del ACV.

El método de evaluación de impacto y caracterización (Eggleston H.S., Buendia L., Miwa K., n.d.) empleado es el CML2016, desarrollado por el Centro de Ciencias Ambientales de la Universidad de Leiden, Holanda quienes publicaron una guía de aplicación de los estándares ISO en la que se establecen categorías de impacto y la correspondiente metodología de caracterización.

El CE se refiere a la cantidad de energía que consume el proceso productivo desde la extracción o, en este caso, el inicio del proceso hasta que está listo para ser comercializado. Incluye también consumo de combustible por transporte. Por otro lado, calentamiento global son las emisiones de GEI que surgen de la producción del APR, en este caso de uso de energía eléctrica. Estas categorías son las más determinantes a la hora de analizar un proceso productivo debido al fuerte efecto que tienen estas variables en el cambio climático que se está viviendo a nivel mundial.

Para las emisiones de GEI se calcula el producto de la cantidad de energía eléctrica consumida $(\mathrm{kW})$ por un factor de caracterización correspondiente a los gases que conforman el CO2 equiv. (CO2, CH4, N2O) Los valores adoptados para los factores de emisión fueron los proporcionados, como se menciona más arriba por CML20016, puesto que en Argentina no se poseen aún datos más confiables (Arena, A.P., Canizo, J. R., Sanchez, n.d.). Luego se suman las contribuciones que cada uno de ellos realiza para calcular los kg equivalentes de CO2.

Los resultados arrojados por la cuantificación de consumos energéticos y correspondientes emisiones de GEI se expresan en la Tabla 2.

\begin{tabular}{|c|c|c|c|c|c|c|}
\hline Equipo & $\begin{array}{c}\text { CE/fact. De } \\
\text { efic.(Kw) }\end{array}$ & CE MJ & CO2 & N20 & CH4 & Kg CO2 Eq) \\
\hline Desgarradora & 573,07 & 2063,04 & 316,58 & 7,30 & 13,81 & 337,68 \\
\hline Molino & 192,7 & 693,93 & 106,49 & 2,45 & 4,64 & 113,58 \\
\hline Mezcladora & 4,82 & 17,35 & 2,66 & 0,06 & 0,12 & 2,84 \\
\hline Total & 770,64 & 2774,32 & 109,15 & 2,52 & 4,76 & 454,11 \\
\hline
\end{tabular}

Tabla 2: consumo energético y sus correspondientes emisiones de GEI

Tanto el consumo energético como su respectiva emisión de GEI, corresponden a menos del 10\% de la emisión de GEI total per cápita en Argentina, o bien, representa la décima parte de lo que emite y consume una persona por año en nuestro País.

Estableciendo comparaciones con trabajos ya publicados sobre agregados naturales/reciclados (ANR) (Mercante et al., 2009)(Wirtz, 2016) y, teniendo en cuenta que factores como la geografía, el transporte, el uso energético, la elección de la UF y método de evaluación afectan en el número final, el consumo energético total para la fabricación de APR corresponde a un 13\% del CE del procesamiento del ANR, al agregar los consumos por transporte a una distancia de la planta de fabricación de APR y de reciclado de RAEE de $15 \mathrm{~km}$ no se producen impactos significativos. El transporte consume de un 0,60 a un $8 \%$ de CAE total por lo que, de encontrarse las plantas emplazadas dentro de 
la misma ciudad, sería el escenario ideal, con un consumo energético de 770 MJ. Si bien, los valores de consumo por transporte son difícilmente comparables debido a que las distancias entre plantas de reciclado o trituración no siempre son las mismas, la geografía de cada lugar con respecto a otro varía significativamente, en este trabajo, incluso con la mayor distancia aplicada entre la planta de reciclado y la planta de fabricación, el consumo energético corresponde a un $0,70 \%$ del consumo del ANR. Con respecto a las emisiones de GEI, el APR emite un total de $780 \mathrm{~kg}$ CO2 equiv. incluyendo las etapas de procesamiento y transporte, mientras que el ANR emite un $20 \%$ más en la totalidad del proceso.

\section{Conclusiones}

Los resultados de los nuevos ensayos se suman a los ya obtenidos con anterioridad concluyendo que las probetas realizadas con APR presentan buenas propiedades mecánicas en general para mampuestos portantes o no, pero deberán ser reforzadas con aditivos o bien con arena gruesa para disminuir la capacidad y velocidad de succión capilar para obtener así valores más cercanos a los que la norma requiere.

El análisis de ciclo de vida demostró que la producción de APR representa un 10\% de las emisiones anuales per cápita en Argentina y, teniendo presentes las limitaciones geográficas, temporales, tecnológicas y metodológicas, presentó un mejor desempeño ambiental que el agregado natural/reciclado fabricado en nuestro país.

\section{Referencias}

Aldrian, A., Ledersteger, A., \& Pomberger, R. (2015). Monitoring of WEEE plastics in regards to brominated flame retardants using handheld XRF. Waste Management, 36, 297-304. https://doi.org/10.1016/j.wasman.2014.10.025

Arena, A.P., Canizo, J. R., Sanchez, B. M. (n.d.). Perfil Ambiental de la generacion termoelectrica en Argentina en el cuartenio 1997-2000.

CARPL. (2010). Estudio sobre los retardantes de llama bromados en los países del Mediterráneo. Centro de Actividad Regional Para La Producción Limpia.

Devia, L. (2012). “Basura electrónica: los riesgos de consumir y descartar,.” Saber Cómo, no. 108, 2.

Dimitrakakis, E., Janz, A., Bilitewski, B., \& Gidarakos, E. (2009). Determination of heavy metals and halogens in plastics from electric and electronic waste. Waste Management, 29(10), 2700-2706. https://doi.org/10.1016/j.wasman.2009.05.020

Eggleston H.S., Buendia L., Miwa K., N. T. y T. K. (eds). (n.d.). IPCC 2006, 2006 IPCC Guidelines for National Greenhouse Gas Inventories, Prepared by the National Greenhouse Gas Inventories Programme. Publicado Por: IGES, Japón., 2.

Global Waste Management Outlook. (2016). In Global Waste Management Outlook. https://doi.org/10.18356/765baec0en

Greenpeace. (2011). El lado toxico de la telefonía movil.

Haarman, A., \& Gasser, M. (2016). Managing hazardous additives in WEEE plastic from the Indian informal sector (Issue June).

Informe, A. y Ta-, S. (2008). Informe final de la Fase I del Proyecto ACAP sobre retardantes de llama bromados (BFR).

Maris, E., Botané, P., Wavrer, P., \& Froelich, D. (2015). Characterizing plastics originating from WEEE: A case study in France. Minerals Engineering, 76, 28-37. https://doi.org/10.1016/j.mineng.2014.12.034

Mercante, I., Arena, P., \& Bovea Edo, M. (2009). Evaluacion De Alternativas De Gestion De Rcd Con Ciclo De Vida. 9.

Schlummer, M., Mäurer, A., Leitner, T., \& Spruzina, W. (2006). Report: Recycling of flame-retarded plastics from waste electric and electronic equipment (WEEE). Waste Management \& Research, 11(4), 287-295., 24(6), 573-583. https://doi.org/10.1177/0734242X06068520

Singh, N., Hui, D., Singh, R., Ahuja, I. P. S., Feo, L., \& Fraternali, F. (2017). Recycling of plastic solid waste: A state of art review and future applications. Composites Part B: Engineering, 115, 409-422. https://doi.org/10.1016/j.compositesb.2016.09.013

Stenvall, E., Tostar, S., Boldizar, A., Foreman, M. R. S. J., \& Möller, K. (2013). An analysis of the composition and metal contamination of plastics from waste electrical and electronic equipment (WEEE). Waste Management, 33(4), 915922. https://doi.org/10.1016/j.wasman.2012.12.022

Tamaddon, F., \& Hogland, W. (1993). Review of Cadmium in Plastic Waste in Sweden. Waste Management \& Research, 11(4), 287-295. https://doi.org/10.1177\%2F0734242X9301100403

Taus, V. L. (2010). Análisis De La Succión Capilar En Hormigones : Influencia De Distintos Parámetros De Ensayo. 193.

Unnisa, S. A., \& Hassanpour, M. (2017). Development circumstances of four recycling industries (used motor oil, acidic sludge, plastic wastes and blown bitumen) in the world. Renewable and Sustainable Energy Reviews, 72(January), 605-624. https://doi.org/10.1016/j.rser.2017.01.109

Wirtz, M. N. (2016). agregado grueso reciclado - propuesta metodológica " Facultad de Ingeniería - Universidad de Buenos Aires. 